\title{
USG Guided Supraclavicular, Vertical Infraclavicular and Coracoid Infraclavicular Approach of Brachial Plexus Block: A Double-Blind Comparative Study
}

\author{
Shah Pratibha Jain ${ }^{1 *}$, Ummu Habeeba ${ }^{2}$, Vijay Kumar ${ }^{3}$ and Mukunda Gowda ${ }^{4}$ \\ ${ }^{1}$ Professor \& HOD, Department of Anaesthesia and Critical Care, Pt JNM MC, Raipur, India \\ ${ }^{2} 3 r d$ Year PG Resident, Department of Anaesthesia and Critical Care, Pt JNM MC, Raipur, India \\ ${ }^{3}$ Specialist grade, The Walton Centre NHS foundation Trust, Liverpool, UK \\ ${ }^{4}$ Senior resident, Department of Anaesthesia and Critical Care, Pt JNM MC, Raipur, India
}

Submission: June 16 2021; Published: October 13, 2021

*Corresponding author: Dr Pratibha Jain Shah, Professor, Department of Anaesthesia and critical care Pt. JNM Medical college, Raipur (C.G.), India, Tel: +919827276620

\begin{abstract}
Background and Aims: Amidst of COVID 19 pandemic, the importance of avoiding aerosol generating procedures (AGP) and doing procedures in regional anaesthesia wherever feasible is of immense value. Brachial plexus block (BPB) is a time tested, valuable and safe alternative to general anaesthesia for upper limb day care surgeries. This randomized double-blind study was conducted to compare three USG guided approaches for BPB using $0.5 \%$ ropivacaine for upper limb surgeries.

Methods: Total 90 patients of ASA grade I- III posted for below mid arm surgeries were randomly allocated into 3 groups (n=30) according to the approach of BPB into Group SC (Supraclavicular approach), Group VI (Vertical infraclavicular approach) \& Group CI (Coracoid infraclavicular approach). Patient comfort, onset, duration \& degree of sensory and motor blockade; duration of complete \& effective analgesia; quality of block and complications related to block were recorded and analyzed.

Results: Patient comfort score 1 was seen in 19 (63.33\%), 20 (66.66\%) \& 28 (93.33\%) patients of SC, VI, CI respectively. Onset \& duration of sensory and motor block was comparable among three groups ( $p>0.05$ ). Duration of complete and effective analgesia was $847.33 \pm 30.16 \mathrm{~min}$ in group SC, $855.66 \pm 36.54 \mathrm{~min}$ in group VI and $856.33 \pm 42.70 \mathrm{~min}$ in group CI, which was similar in all three groups. There was significant decrease in complication with CI compared to other groups $(\mathrm{p}<0.05)$ of which pneumothorax was not seen in Group CI while $2(6.66 \%)$ and $1(3.33 \%)$ patients were noted in Group SC and VI, respectively.

Conclusion: Though all the three approaches of BPB are equally effective in terms of block characteristics, $\mathrm{CI}$ approach is better and could be preferred for below middle arm surgeries due to low complication rate and patient comfort.

Keywords: Brachial plexus block, supraclavicular approach, vertical infraclavicular approach, coracoid infraclavicular approach, USG, ropivacaine
\end{abstract}

\section{Introduction}

The role played by USG is pivotal to partake higher success in regional anaesthesia, thereby avoiding the requirement of supplemental anaesthesia, general anaesthesia (GA) or other AGPs in this COVID-19 pandemic era [1].

BPB gives us a fully conscious patient, avoids polypharmacy, and provides better hemodynamic stability as well as an excellent post-operative analgesia [2]. Supraclavicular approach (SC) is most popular owing to high success rate and quick onset from tightly grouped nerves, which is superficial and easily visible under ultrasound, but complications like inadvertent intravenous injection of drugs, pneumothorax, phrenic nerve palsy and Horner's syndrome are more [2-4]. Despite of its remunerations of less complications \& more consistent technique, clinical use of infraclavicular block (ICb) is not widespread [5]. This might be due to uncertain surface landmarks, and the discernment that ICbs are more painful.

Selection of the approach is usually determined by the surgical site, preference \& experience of the anaesthesiologist, reliability, ease, rapidity \& patient comfort during block performance and risk of complications. 
There are very few studies that compares USG guided supraclavicular (SC), vertical infraclavicular (VI) and coracoid infraclavicular (CI) approach of BPB together. Therefore, this present prospective, double-blind randomized study was conducted to find out best approach amongst three in terms of patient comfort as a primary outcome and characteristics of sensory and motor blockade (onset, duration, and degree), duration of complete \& effective analgesia, quality of block and complications related to block as secondary outcomes.

\section{Patients and Method}

After obtaining approval from institutional scientific \& ethics committee and written informed consent from patients, present study was conducted according to Helsinki 2 declaration in total of 90 patients of ASA I-III, 18-50 years age, and 50-75 kg body weight who were posted for elective below mid arm surgeries from June 2018- August 2019. Those with incapability for consent or refused to enrol, infection/ ulcer at the site of needle insertion, coagulopathy, contralateral phrenic \& recurrent laryngeal nerve paralysis, chest deformities, pneumothorax, significant pulmonary pathology, pregnancy and known history of allergy to local anaesthetic were excluded from the study.

Sample size was calculated based on Abhinaya et al. study [6]. For the study to have $80 \%$ power and alpha error at 0.05 , a minimum of 28 patients would be required in each group to detect a $20 \%$ difference in assessment of quality of block, assuming a standard deviation of 1.5 .

All participants were kept nil per oral for 6 hours prior to operation. Patients were shifted to block performing room after thorough pre-anaesthetic check-up. Baseline Heart Rate (HR), Systolic Blood Pressure (SBP), Diastolic Blood Pressure (DBP) and Oxygen Saturation (SpO2) on room air were recorded. Intravenous (IV) line was accessed by inserting $18 \mathrm{G}$ cannula. All the patients were premedicated with iv midazolam $0.02 \mathrm{mg} / \mathrm{kg}$ and received iv ringer lactate at $5-6 \mathrm{ml} / \mathrm{kg} / \mathrm{h}$. BPB was performed as per group allocated using sequentially numbered opaque sealed envelope technique (SNOSE).

Block was performed in supine position with head turned away from the side to be blocked under strict aseptic precautions after intradermal anaesthesia with $1 \mathrm{ml}$ of $2 \%$ lignocaine at entry point of approach for BPB using USG machine (Mindray 3C5s) with $12 \mathrm{HZ}$ frequency linear probe. The objective was to place the needle in the brachial plexus sheath and inject ropivacaine to visualize the spread within the brachial plexus and the centrifugal displacement of the trunks, divisions and cords. If 10 min lapsed without obtaining adequate image of the target, approach was halted, and patient was excluded from further assessment. All the blocks were performed by senior anesthesiologist trained in USG guided block. The data were recorded and analyzed by the assistant who was blinded to the approach used for BPB.

For SC approach, the ultrasound probe was placed in the supraclavicular fossa under sterile conditions then subclavian artery, pleura and first rib were recognised. Trunks / division were identified as "grape like" structures and a 22-gauge $50 \mathrm{~mm}$ needle was introduced using an in-plane technique into the brachial plexus sheath and $20 \mathrm{ml} 0.5 \%$ ropivacaine was pushed in $5 \mathrm{ml}$ increments after negative aspirations and appreciating centrifugal displacement of trunks \& divisions on the screen.

For VI approach, the USG probe was placed under aseptic precaution in the infraclavicular fossa between the sternoclavicular joint and acromion process to visualize axillary artery. Using an in-plane technique, 22-gauge $50 \mathrm{~mm}$ needle was introduced in a parasagittal plane until the tip was located just posterior to the axillary artery and $20 \mathrm{ml}$ of $0.5 \%$ ropivacaine was injected in a U-shaped manner around axillary artery in $5 \mathrm{ml}$ increments after negative aspiration.

For CI approach, patient had the full freedom to keep their painful arms in adduction or abduction. Linear probe was kept medial and inferior to the coracoid process in deltopectoral groove to view axillary vessels and cords in short axis. The lungs and pleura were visualized, and the needle direction was kept away from that. A 22-gauge $50 \mathrm{~mm}$ needle is introduced parallel \& in plane to the probe. The horizontal trajectory of the needle was traced and $20 \mathrm{ml}$ of $0.5 \%$ ropivacaine was injected as $5 \mathrm{ml}$ small aliquots with negative aspirations in a U-shaped manner around the axillary artery.

To assess the patient comfort, patients were asked for their satisfaction level during the performance of block and surgery by the two-point assessment scale 0 -unsatisfied and 1-satisfied [7]. For this, all patients were contacted within $48 \mathrm{~h}$ of the surgery and enquired about the complications like chest pain, breathing difficulty, bruises, swelling over the area of block and their preferences for future upper limb surgeries i.e., the same block again or General Anaesthesia. Pain during block performance was also gauged using VAS score on a scale of 0 to 10 ; where 0 - no pain and 10 - Severe agonizing pain.

The onset \& degree of sensory and motor block were observed in every $5 \mathrm{~min}$ for $30 \mathrm{~min}$ till complete blockade was achieved. Block was considered "failed" if patient perceived pain after 30 min of blockade, required local infiltration or converted into GA. Assessment of block was done by sensory loss over the cutaneous innervation territories of radial nerve (lateral aspect of the dorsum of the hand), median nerve (volar aspect of the thumb), ulnar nerve (volar aspect of the fifth finger), musculocutaneous nerve (lateral aspect of forearm) and medial cutaneous nerve of forearm (medial aspect of forearm). Sensory score was graded as follows, Grade 0: No difference from unblocked extremity, Grade 1: Less cold from unblocked extremity, Grade 2: No sensation of cold. 5 Motor blocks was assessed using 0- normal strength, 1- weakness and 2- paralysis for the following nerves including radial nerve (thumb abduction), median nerve (thumb opposition), ulnar nerve (thumb adduction) and musculocutaneous nerve (elbow flexion) [8]. 
Time of ropivacaine injection, achievement of grade 2 sensory \& motor blockade, return of blockade to grade 0 and injection of first rescue analgesic i.e., paracetamol infusion $1 \mathrm{gm}$ were recorded. From those parameters, onset and duration of sensory \& motor blockade along with effective analgesia were calculated. The onset of sensory and motor blockade was considered from the time of administration of the ropivacaine to the time of complete sensory blockade up to grade 2 and the time of complete motor blockade unto grade 2, respectively. The duration of sensory \& motor blockade was calculated from onset to return of sensory \& motor blockade to grade 0 , respectively. The duration of the effective analgesia was defined as the time gap between instillation of ropivacaine to the administration of first rescue analgesic in our study.

Haemodynamic parameters (HR, SBP, DBP, MBP, RR and SpO2) were monitored initially at 5 minutes intervals for 30 minutes and after that at 10 minutes intervals for the entire duration of surgery. and side effects (accidental vascular puncture, pneumothorax, diaphragmatic paresis, Horner's syndrome, and hematoma) were also noted. A post block chest x-ray was obtained if patient complains of respiratory distress.

Ninety patients were recruited in this study and a Consolidated Standards of Reporting Trials flow diagram illustrating the passage of participants through the study has been given in Figure 1.

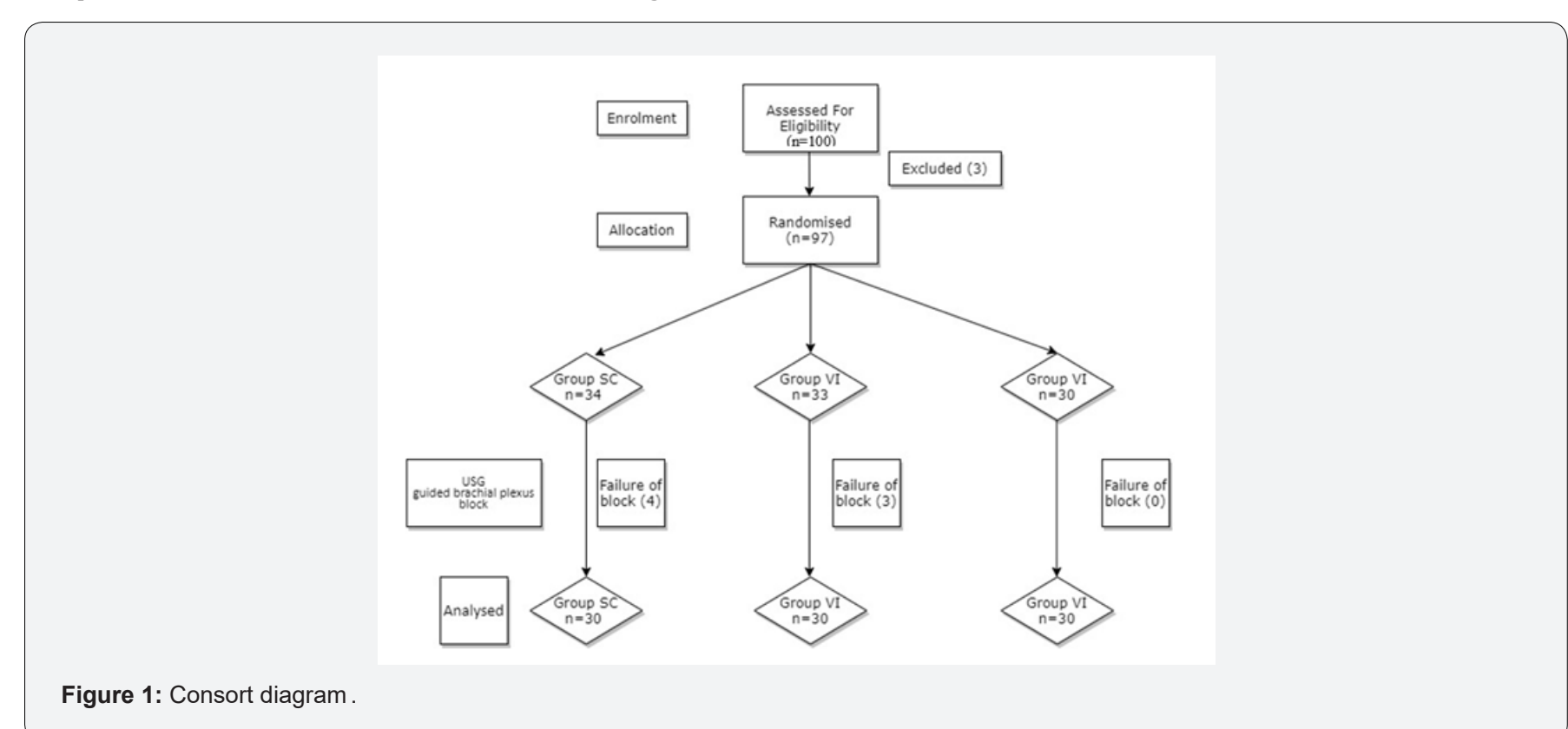

Statistical analysis was performed using Graph pad in stat software. Results were expressed as mean $\pm \mathrm{SD}$, number, and percentage (\%). Proportions were analyzed using the ANOVA test. All tests were two tailed and performed at a significance level of 0.05 .

\section{Results}

All the groups were statistically comparable in terms of demographic data profile and duration of surgery. (Table 1) The data obtained were not skewed.

Table 1: Demographic profile and duration of surgery.

\begin{tabular}{|c|c|c|c|c|}
\hline Item & Group SC & Group VI & Group Cl & $\boldsymbol{p}$ \\
\hline $\begin{array}{c}\text { Age } \\
(\mathrm{yr})\end{array}$ & $24.15 \pm 9.34$ & $25.32 \pm 7.86$ & $23.42 \pm 8.41$ & $0.614^{*}$ \\
\hline $\begin{array}{c}\text { Height } \\
(\mathrm{cm})\end{array}$ & $168 \pm 3.45$ & $170 \pm 2.36$ & $166 \pm 5.32$ & $0.096^{*}$ \\
\hline $\begin{array}{c}\text { Weight } \\
(\mathrm{kg})\end{array}$ & $68 \pm 7.12$ & $70 \pm 8.47$ & $71 \pm 7.48$ & $0.725^{*}$ \\
\hline $\begin{array}{c}\text { Duration of } \\
\text { Surgery (min) }\end{array}$ & $65 \pm 5.41$ & $70 \pm 3.42$ & $58 \pm 7.86$ & $0.563^{*}$ \\
\hline
\end{tabular}


Maximum patients were comfortable, had score 1 while putting block in all the three groups, but it was significantly high in Group CI (93.33\%) when compared to SC (63.3\%) \& VI (66.7\%)
(Figure 2). The technique related pain score (VAS score) was also statistically comparable amongst the studied groups (SC- $2.54 \pm$ 0.35 , CI $-2.13 \pm 0.21 \& \mathrm{VI}-2.74 \pm 0.72$ ).

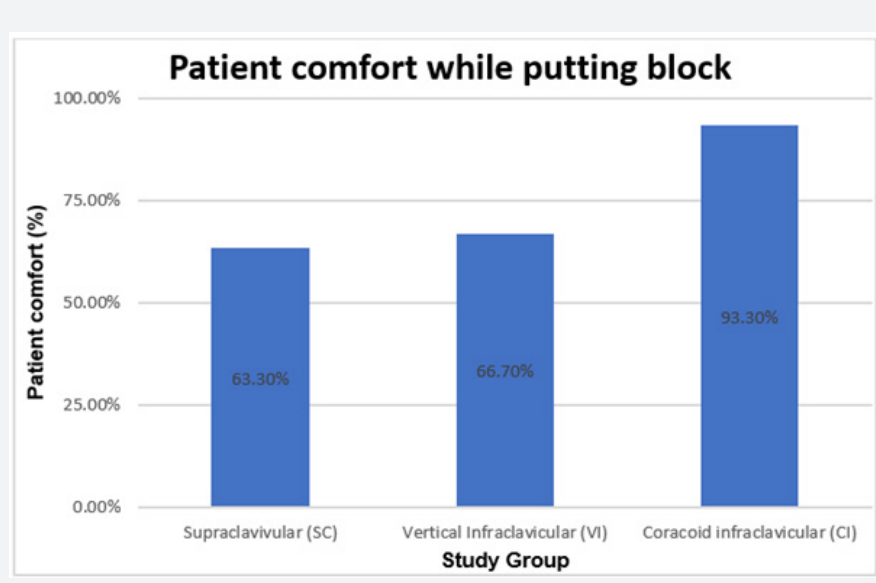

Figure 2: Patient comfort (Score 1) while putting block.

The time of onset and duration of sensory \& motor blockade were statistically comparable among three groups. ( $\mathrm{p}=0.081$ \& 0.094 and $0.382 \& 0.851$, respectively) Duration of effective

Table 2: Block characteristics. analgesia was also statistically comparable among three groups. $(\mathrm{p}=0.575)$ (Table 2)

\begin{tabular}{|c|c|c|c|c|c|}
\hline S NO. & Block characteristics & SC & VI & CI & P value \\
\hline 1 & Onset of Sensory Blockade & $13.71 \pm 1.006$ & $13.3 \pm 0.664$ & $13.3 \pm 0.702$ & 0.081 \\
\hline 2 & Onset of Motor Blockade & $19.50 \pm 0.787$ & $19.63 \pm 0.718$ & $19.76 \pm 0.653$ & 0.382 \\
\hline 3 & Duration of Sensory Blockade & $794.76 \pm 32.00$ & $781.66 \pm 16.62$ & $783.33 \pm 24.11$ & 0.094 \\
\hline 4 & Duration of Motor Blockade & $807.66 \pm 32.12$ & $802.73 \pm 35.34$ & $304.33 \pm 35.20$ & 0.851 \\
\hline 5 & Duration of Effective Analgesia & $847.33 \pm 30.16$ & $855.66 \pm 36.54$ & $856.33 \pm 42.70$ & 0.575 \\
\hline
\end{tabular}

The vital parameters like heart rate, systolic and diastolic blood pressure were comparable among three groups throughout

the study period (Figures $3 \& 4$ ).

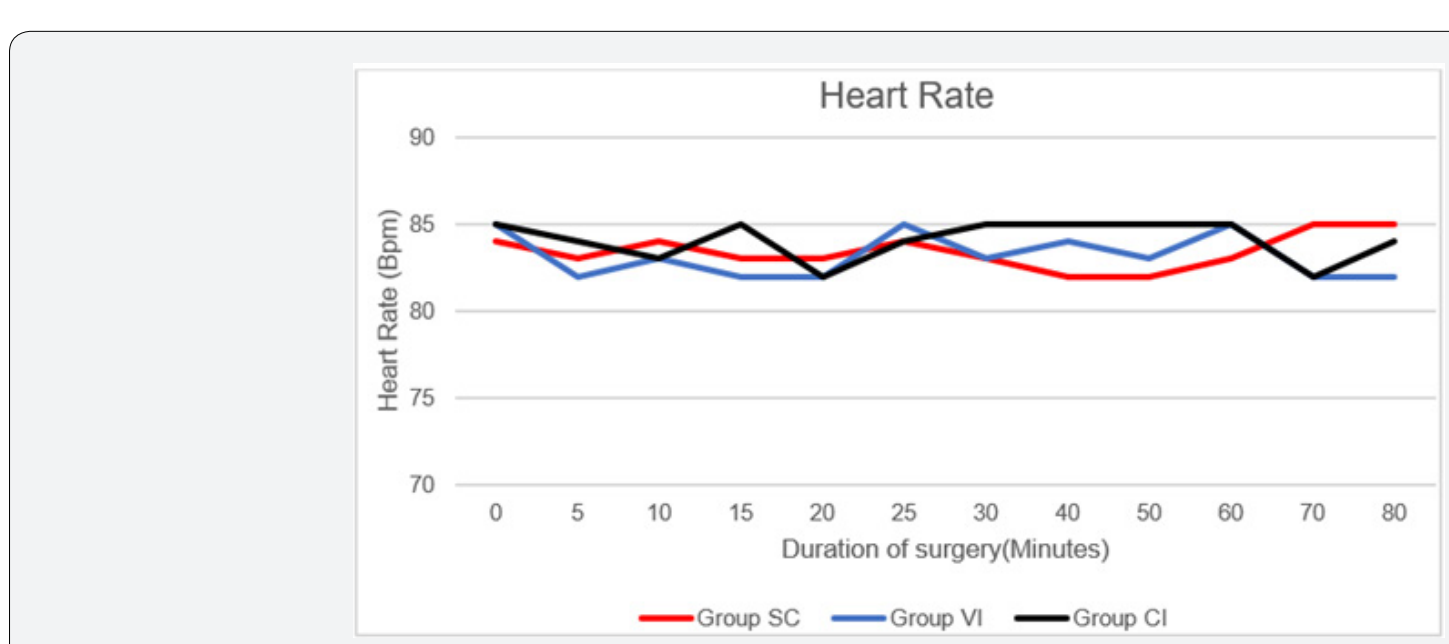

Figure 3: Changes in heart rate during surgery. 


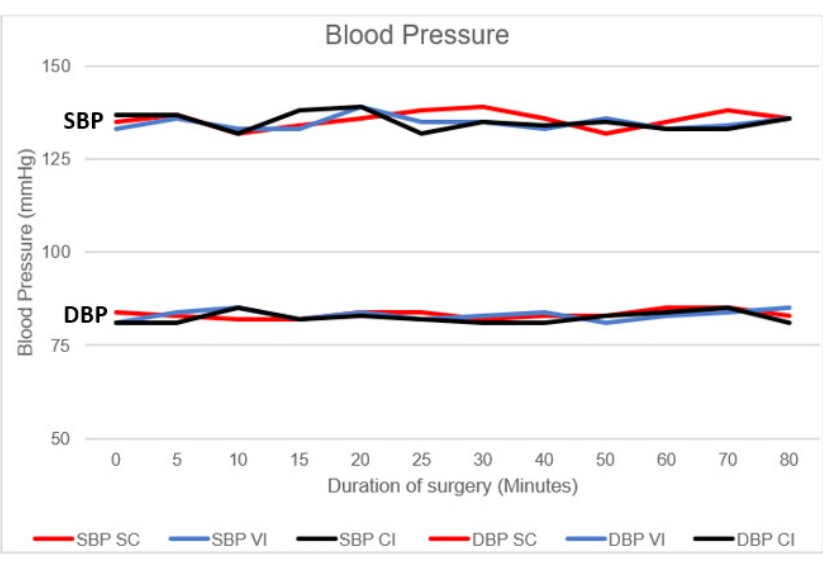

Figure 4: Changes in SBP and DBP during surgery.

Overall complications were significantly low in Group CI compared to Group SC and VI. Pneumothorax was not seen in Group CI, while 6.66\% patients in Group SC and 3.33\% patients in Group VI had pneumothorax. Similarly, low incidence of vascular puncture was noted in Group CI (3.33\%) compared to Group SC and Group VI. Incidence of hematoma was 3.33\% in SC as well as in VI but not observed in CI Groups. Incidence of dyspnoea was also significantly low in Group CI compared to SC group (Figure 5).

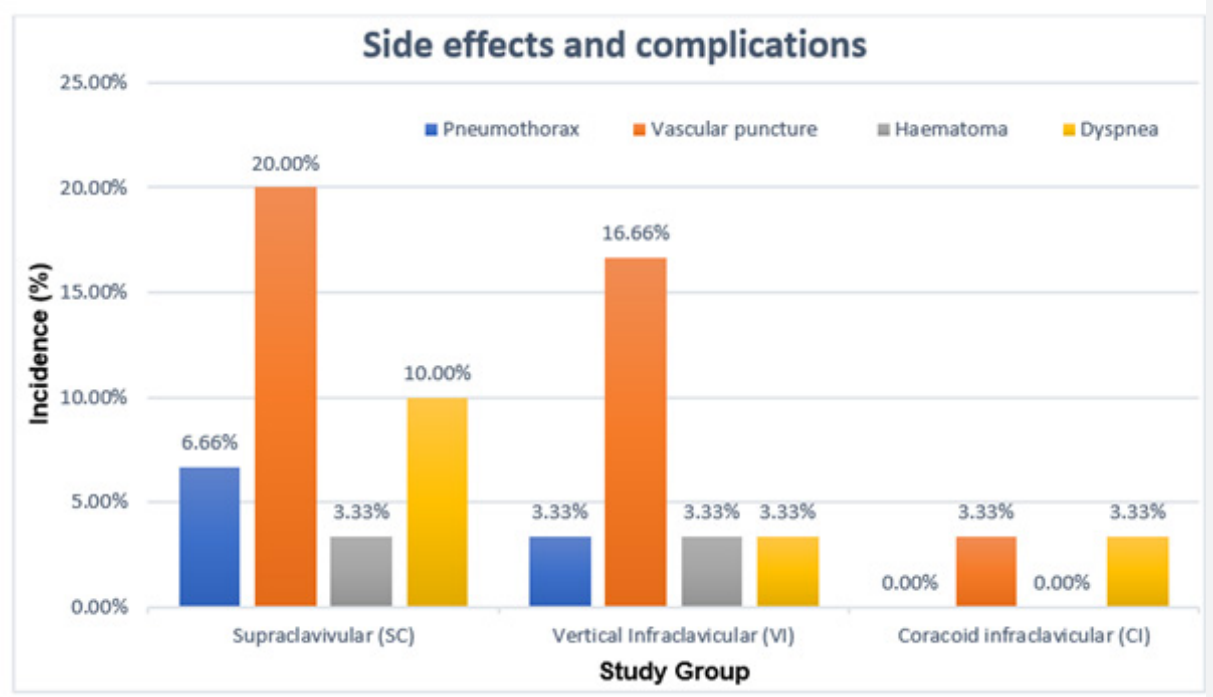

Figure 5: Side effects and complications.

\section{DISCUSSION:}

The results in this study showed that the coracoid approach of brachial plexus is grander to vertical infraclavicular and the supraclavicular approach in handling upper limb surgeries due to better patient comfort and lesser complications.

In our study, patient comfort was testified to be score 1 in $93.3 \%$ of CI compared to $66.7 \%$ \& $63.3 \%$ in VI and SC groups, respectively. Arcand et al. [9]. and Neilson et al. [10]. also observed better patient satisfaction with infraclavicular approach than supraclavicular approach. Trehan et al. [5]. also noted better patient satisfaction with coracoid infraclavicular approach than vertical infraclavicular approach. In the study performed by Abhinaya et al. [6]. the patient satisfaction score was reported higher in infraclavicular approach (93.3\%) compared to $90 \%$ in supraclavicular approach of BPB, but it was not statistically significant $(\mathrm{p}=0.640)$. This higher degree of satisfaction might be due to utilization of both ultrasound and nerve stimulator in their study that favor the comfortable position of patients in all approaches of BPB.

Pain score while performing block was comparable amongst the 3 groups in our study which was alike the observation by Arcand et al. [9]. 
The time of onset of sensory and motor blockade was comparable amongst the three approaches of BPB studied using ropivacaine in our study. In Tawfic $\mathrm{T}$ A et al study [11]., the onset of block was $14.01 \mathrm{~min}$ in ropivacaine group (30 $\mathrm{ml}, 0.25 \%$ ) and $8.65 \mathrm{~min}$ in bupivacaine group ( $30 \mathrm{ml}, 0.25 \%)$. This coincides with the results of our study. Abhinaya et al [6]. reported a significantly faster onset of sensory and motor blockade, $6.43 \pm 2.61 \mathrm{~min}$ and $7.32 \pm 2.90 \mathrm{~min}$ in infraclavicular group \& $8.45 \pm 2.87 \mathrm{~min}$ and $8.68 \pm 3.50 \mathrm{~min}$ in supraclavicular group, respectively. This early onset in sensory and motor blockade might be due to larger volume of local anaesthetic ( $30 \mathrm{ml} 0.5 \%$ ropivacaine) instillation in their study under the guidance of both USG and nerve stimulator [6]. This difference may also be due to different definitions of onset time, differences in local anaesthetic agents used and the difference in the concentration used.

Duration of sensory and motor blockade was also statistically comparable amongst three approaches studied in our study. Our results were analogous to Yang et al. study.[7]. Duration of sensory and motor blockade was not accounted for in the study conducted by Abhinaya et al. [6].

Duration of effective analgesia was observed to be $847.33 \pm 30.16 \mathrm{~min}$ in $\mathrm{SC}, 855.66 \pm 36.54 \mathrm{~min}$ in group VI and $856.33 \pm 42.70 \mathrm{~min}$ in group CI, respectively. In Arcand et al [9]. study, the duration of effective analgesia was $436 \pm 167 \mathrm{~min}$ and $471 \pm 215 \mathrm{~min}$, respectively for IC and SC. The decrement of duration in their study may be due to the usage of different drug, i.e., mixture of $0.5 \%$ bupivacaine and $2 \%$ lignocaine with adrenaline 1:200,000 having larger proportion of lignocaine.

Overall, incidence of complications were lower in the IC approach, especially in the CI approach in present study. This was like Trehan et al. [5]. study where they noted lower incidences of complications in both approaches of infraclavicular block (.). In our study, the vascular puncture was noted in $3.33 \%$ patients with CI approach, $16.66 \%$ with VI approach and $20 \%$ in SC approach. Yang et al. [7]. observed $16 \%$ of vascular puncture in supraclavicular group and $14 \%$ in infraclavicular group. Pneumothorax was nil with CI, 3.3\% with VI and $6.66 \%$ with SC, in our study. Similarly, there was a $4 \%$ incidence of pneumothorax with supraclavicular approach compared to zero incidences with infraclavicular approach in the study conducted by Yang et al. [7]. This might be due to needle direction, which is away from the pleura unlike in the supraclavicular approach [12]. Trehan et al [5]. also got zero incidences of pneumothorax in both approaches of infraclavicular BPB. The incidence of dyspnea was 3.33\% in both CI \& VI group and was $10 \%$ in SC group in our study. Petrar SD et al [15] noted dyspnea in $25 \%$ of the USG guided supraclavicular group versus $16 \%$ of the USG guided infraclavicular group. This might be due to the fact that spread of local anesthetic involving phrenic nerve which causes hemi diaphragmatic paralysis. Yang et al. [7]. reported $6 \%$ incidence of dyspnoea with supraclavicular approach when compared to zero incidences in infraclavicular approach. There were no such incidences in CI and VI approaches of infraclavicular block in Trehan et al. [5]. study. The hematoma was not in any patient in CI group compared to $3.33 \%$ in other 2 groups in our study. The hematoma was seen in $8.1 \%$ and $4.6 \%$ in the supraclavicular and infraclavicular approach in the study conducted by Kilic et al. study [13]. All the patients who developed complications or side effects were managed conservatively and were discharged after 24 hours of observations.

Hence, these findings endorse our hypothesis that coracoid infraclavicular approach of BPB is better in terms of patient comfort and lesser complications when compared to the other two approaches under USG guidance. Although extrapolating our observation need a large sample size, the major limitation of our study [14].

In the future, the same study can be protracted with the utilization of catheter placement for better post-operative analgesia. We can also seek a lower dose of local anaesthetic under ultrasound guidance in near-term studies.

\section{Conclusion}

Though, supraclavicular, vertical infraclavicular and coracoid infraclavicular approaches of brachial plexus block were effectual in terms of onset, duration and effectiveness of sensory \& motor blockade for below mid-arm surgeries; coracoid infraclavicular brachial plexus approach is comparatively prudent with reference to patient satisfaction and less complications.

\section{Conflicts of Interest}

There are no conflicts of interest.

\section{References}

1. Ponde VC, Diwan S, Gopal TV, Subramanian J B, Danish MA (2020) Regional anaesthesia in the coronavirus disease (COVID-19) pandemic: Clinical guidelines by AORA, India. J Anaesthesiol Clin Pharmacol 36(Suppl 1): S109-S115.

2. Tran DQ Russo G, Muñoz L, Zaouter C, Finlayson RJ (2009) A prospective, randomized comparison between ultrasound-guided supraclavicular, infraclavicular, and axillary brachial plexus blocks. Reg Anesth Pain Med 34(4): 366-371.

3. Stav A, Reytman L, Stav M-Y, Portnoy I, Kantarovsky A, et al. (2016) Comparison of the Supraclavicular, Infraclavicular and Axillary Approaches for Ultrasound-Guided Brachial Plexus Block for Surgical Anesthesia. Rambam Maimonides Med J 7(2): e0013.

4. Prasad PK, Lakshmi BS, Sophia P, Sirisha V (2014) Comparison of Lateral Approach with Conventional Approach of Supraclavicular Brachial Plexus Block: A Prospective Randomized Comparative Study. Journal of Evolution of Medical and Dental Sciences 3: 8661-8669.

5. Trehan V, Srivastava U, Kumar A, Saxena S, Singh CS, et al. (2010) Comparison of two approaches of infraclavicular brachial plexus block for orthopaedic surgery below mid-humerus. Indian J Anaesth 54(3): 210-241.

6. Abhinaya RJ, Venkatraman R, Matheswaran P, Sivarajan G (2017) A randomised comparative evaluation of supraclavicular and infraclavicular approaches to brachial plexus block for upper limb surgeries using both ultrasound and nerve stimulator. Indian J Anaesth. 2017 Jul; 61(7): 581-586. 
7. Yang CW, Kwon HU, Cho CK, Jung SM, Kang P-S, et al. (2010) A comparison of infraclavicular and supraclavicular approaches to the brachial plexus using neurostimulation. Korean J Anesthesiol 58(3): 260-266.

8. Sinha C, Kumar N, Kumar A, Kumar A, Kumar A (2019) Comparative evaluation of two approaches of infraclavicular brachial plexus block for upper-limb surgeries. Saudi J Anaesth 13(1): 35-39.

9. Arcand G, Williams SR, Chouinard P, Boudreault D, Harris P, et al. (2005) Ultrasound-guided infraclavicular versus supraclavicular block. Anesth Analg 101(3): 886-890

10. Koscielniak-Nielsen ZJ, Frederiksen BS, Rasmussen H, Hesselbjerg L (2009) A comparison of ultrasound-guided supraclavicular and infraclavicular blocks for upper extremity surgery. Acta Anaesthesiol Scand 53(5): 620-626.

11. Tawfic TA, Agameya HM (2006) A clinical and pharmacokinetic comparison of ropivacaine and bupivacaine for supraclavicular brachial plexus block in patients with chronic renal failure. Alex Anaesth Intensive Care 9(2): 23-28.
12. Lahori VU, Raina A, Gulati S, Kumar D, Gupta SD (2011) A randomized comparative study of efficacy of axillary and infraclavicular approaches for brachial plexus block for upper limb surgery using peripheral nerve stimulator. Indian J Anaesth 55(3): 253-259.

13. Kılıç ET, Akdemir MS (2018) Comparison of Supraclavicular Infraclavicular, and Axillary Approaches for Ultrasound-Guided Brachial Plexus Block for Upper Limb Surgeries: A Retrospective Analysis of 182 Blocks. Dubai Med J 1: 33-37.

14. Markus Z, Philipp O, Peter M, Anna I, Thomas H (2020) Brachial plexus block with ultrasound guidance for upper-limb trauma surgery in children: a retrospective cohort study of 565 cases. Br J Anaesth 125 (1): $104 \mathrm{e} 109$.

15. Petrar SD, Seltenrich ME, Head SJ, Schwarz SKW (2015) Hemidiaphragmatic Paralysis Following Ultrasound-Guided Supraclavicular Versus Infraclavicular Brachial Plexus Blockade: A Randomized Clinical Trial. Reg Anesth Pain Med 40(2): 133-138.
Your next submission with Juniper Publishers will reach you the below assets

- Quality Editorial service

- Swift Peer Review

- Reprints availability

- E-prints Service

- Manuscript Podcast for convenient understanding

- Global attainment for your research

- Manuscript accessibility in different formats

( Pdf, E-pub, Full Text, Audio)

- Unceasing customer service

Track the below URL for one-step submission https://juniperpublishers.com/online-submission.php 